DRAFT VERSION JANUARY 7, 2020

Typeset using $\mathrm{LAT}_{\mathrm{E}} \mathrm{X}$ preprint2 style in AASTeX63

\title{
Onset of Cosmic Reionization: Evidence of An Ionized Bubble Merely 680 Myrs after the Big Bang
}

\author{
V. Tilvi, ${ }^{1}$ S. Malhotra, ${ }^{2}$ J. E. Rhohds, ${ }^{2}$ A. Coughlin,${ }^{3}$ Z. Zheng, ${ }^{4}$ S. L. Finkelstein ${ }^{5}$ \\ S. Veilleux,${ }^{6}$ B. Mobasher,${ }^{7}$ J. Wang,${ }^{8}$ R. Probst,${ }^{9}$ R. Swaters, ${ }^{6}$ P. Hibon,${ }^{10}$ B. Joshi,${ }^{1}$ \\ J. ZABL, ${ }^{11}$ T. JIANG, ${ }^{1}$ J. PhARO, ${ }^{1}$ AND H. YANG ${ }^{1}$ \\ ${ }^{1}$ School of Earth and Space Exploration, Arizona State University, Tempe, AZ 85287, USA \\ ${ }^{2}$ Astrophysics Science Division, Goddard Space Flight Center, Greenbelt, MD 20771, USA \\ ${ }^{3}$ Chandler-Gilbert Community College, Chandler, AZ 85225-2499 USA \\ ${ }^{4}$ CAS Key Laboratory for Research in Galaxies and Cosmology, Shanghai Astronomical Observatory, Shanghai \\ 200030, People's Republic of China \\ ${ }^{5}$ Department of Astronomy, The University of Texas at Austin, Austin, TX 78712, USA \\ ${ }^{6}$ Department of Astronomy and Joint Space-Science Institute, University of Maryland, College Park, MD 20742, USA \\ ${ }^{7}$ Department of Physics and Astronomy, University of California, Riverside, CA 92521 USA \\ ${ }^{8}$ CAS Key Laboratory for Research in Galaxies and Cosmology, Department of Astronomy, University of Science and \\ Technology of China, Hefei, Anhui 230026, People's Republic of China \\ ${ }^{9}$ NOAO, 950 N. Cherry Avenue, Tucson, AZ 85719, USA \\ ${ }^{10}$ European Southern Observatory, Alonso de Cordova 3107, Casilla 19001, Santiago, Chile \\ ${ }^{11}$ Univ Lyon, Univ Lyon1, Ens de Lyon, CNRS, Centre de Recherche Astrophysique de Lyon UMR5574, F-69230
} Saint-Genis-Laval, France

\begin{abstract}
While most of the inter-galactic medium (IGM) today is permeated by ionized hydrogen, it was largely filled with neutral hydrogen for the first 700 million years after the Big Bang. The process that ionized the IGM (cosmic reionization) is expected to be spatially inhomogeneous, with fainter galaxies playing a significant role. However, we still have only a few direct constraints on the reionization process. Here we report the first spectroscopic confirmation of two galaxies and very likely a third galaxy in a group (hereafter EGS77) at redshift $z=7.7$, merely 680 Myrs after the Big Bang. The physical separation among the three members is $<0.7 \mathrm{Mpc}$. We estimate the radius of ionized bubble of the brightest galaxy to be about $1.02 \mathrm{Mpc}$, and show that the individual ionized bubbles formed by all three galaxies likely overlap significantly, forming a large yet localized ionized region, which leads to the spatial inhomogeneity in the reionization process. It is striking that two of three galaxies in EGS77 are quite faint in the continuum, thanks to our selection of reionizing sources using their Lyman- $\alpha$ line emission. Indeed, one is the faintest spectroscopically confirmed galaxy yet discovered at such high redshifts. Our observations provide direct constraints in the process of cosmic reionization, and allow us to investigate the properties of sources responsible for reionizing the universe.
\end{abstract}

Corresponding author: V. Tilvi

tilvi@asu.edu 
Keywords: editorials, notices — miscellaneous — catalogs — surveys

\section{INTRODUCTION}

Cosmological simulations indicate that the process of reionization, which is expected to be patchy or spatially inhomogeneous (Furlanetto et al 2004, Iliev et al. 2006, Zahn et al. 2007, Mesinger A. et al. 2008, Jensen, H. et al 2014), started when intense UV radiation from individual galaxies or groups of galaxies first ionized their local surroundings, and formed local ionized bubbles. These ionized bubbles later grew to fill the entire IGM, marking the end of the reionization process.

Star-forming galaxies at high redshifts are expected to have contributed to the reionization process (e.g. Stiavelli et al. 2004; Bouwens et al. 2015). These same galaxies, via their Lyman- $\alpha$ emission, provide a practical tool to study the reionization process - the evolution of neutral hydrogen in the IGM. Currently however, we lack a clear observational evidence of the spatial inhomogeneity or the sources responsible for the cosmic reionization. Therefore, to study the nature of cosmic reionization, and quantify galaxies' contribution to this process, we have carried out a unique narrow-band (NB) imaging survey, the Cosmic Deep And Wide Narrowband survey (DAWN; PI: Rhoads) to observe Lyman$\alpha$ emitting galaxies at high redshifts. The NB technique has been proven successful in identifying $z>6$ galaxies (Hu et al. 2010; Ouchi et al. 2010; Rhoads et al. 2012) which otherwise could go undetected in the traditional broad-band selection techniques (e.g. Steidel et al. 2003). Here we present discovery of the most distant galaxy group, identified using NB imaging, and confirmed via spectroscopic observations.

\section{IMAGING}

\subsection{Observations \& Data Reduction}

We obtained deep NB imaging observations of the Extended Groth Strip (EGS) field (RA 14:19:16 DEC +52:52:13), as part of the DAWN survey. This is a uniquely deep survey given its sensitivity as well as area coverage, with a primary objective of identifying galaxies at redshift $z=7.7$. Here we present relevant details of the DAWN survey (Rhoads et al in prep, Coughlin et al 2018). The DAWN survey was carried out using a custom built narrow-band filter $(\mathrm{FWHM}=35 \AA$, central wavelength $=10660 \AA)$, mounted on the NOAO Extremely Wide-Field InfraRed Imager (NEWFIRM)(Probst et al. 2004, 2008) at the Kitt Peak 4m Mayall telescope. The NEWFIRM instrument has a wide field of view $\left(28 \times 28 \operatorname{arcmin}^{2}\right)$ with a resolution of $0.4^{\prime \prime}$ per pixel. We obtained individual images with 600s integration time and Fowler samples of 16 with 8 digital averages during the readout. To achieve clean sky background subtraction we used random dithering with a dither size of $45^{\prime \prime}$.

The data reduction was primarily done using the NEWFIRM science data reduction pipeline (Swaters et al. 2009). However, for generating the final stack of all the images produced by the pipeline (sky subtracted, cosmicrays cleaned, re-projected) we used our own scripts to remove bad frames that were visually inspected in order to maximize the signalto-noise ratio of astronomical objects. The final NB stack is equivalent to a total integration time of $67 \mathrm{hrs}$, yielding $5 \sigma$ line flux sensitivity of $\sim 7 \times 10^{-18} \mathrm{erg} \mathrm{s}^{-1} \mathrm{~cm}^{-2}$. In addition to the NB image described above, to select high-redshift Lyman- $\alpha$ emission line candidates, we also used publicly available broadband images at visible wavelengths (HST/ACS F606W, F814W) and at near-IR wavelengths (HST/WFC3 F125W, F160W). The observations at visible wavelengths were taken as part of the GO10134 program (PI: M. Davis). Near- 

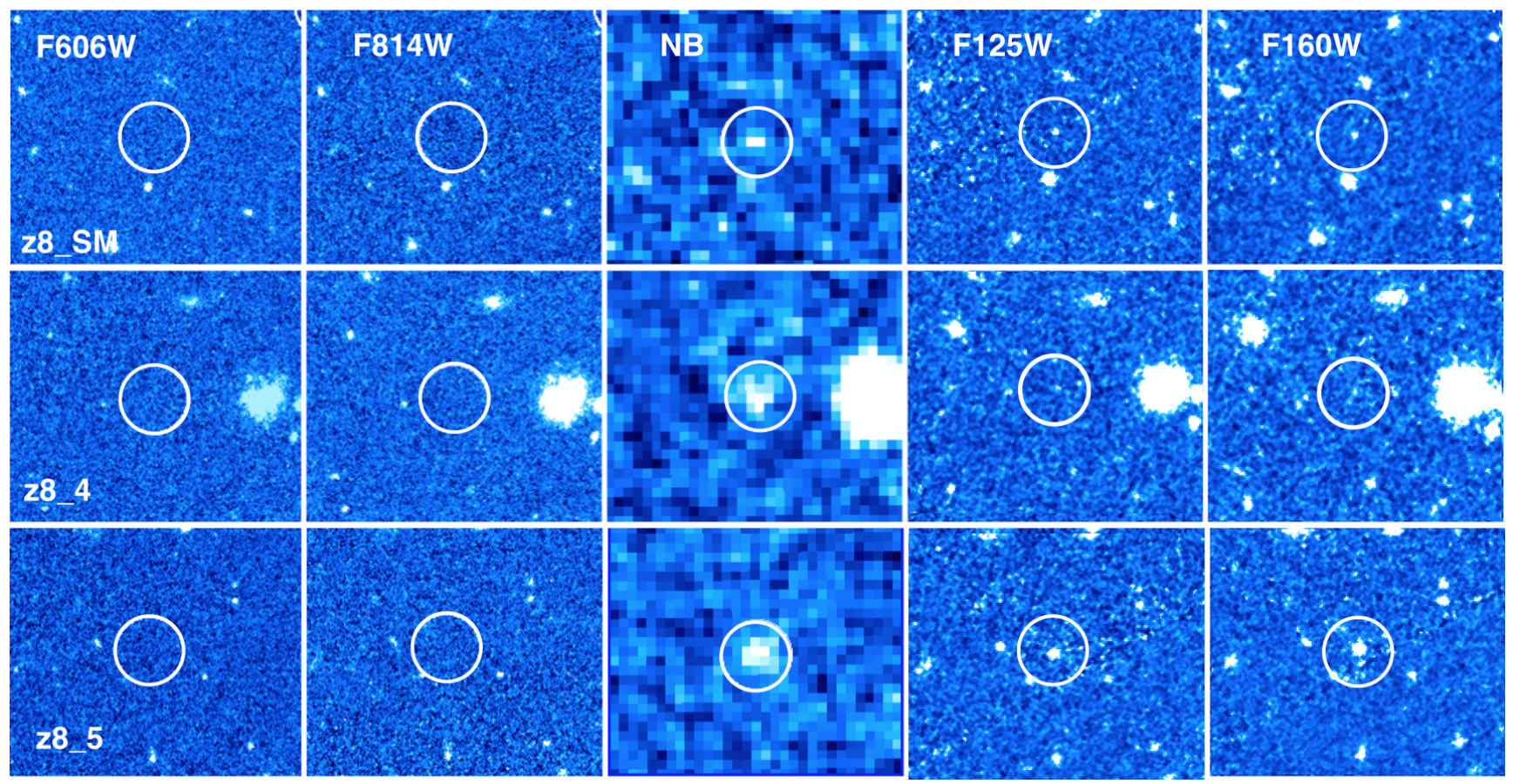

Figure 1. Image cutouts of all three members of EGS77. All galaxies are detected at redder wavelengths (NB, F125W, and F160W), while undetected at the visible wavelengths (F606W and F814W). The sharp drop in the flux at visible wavelengths is consistent with the objects being at redshifts $z \geq 7$. All cutouts are $\sim 12^{\prime \prime}$ on the side, and the circles enclosing objects have $1.4^{\prime \prime}$ radius. NB images appear pixelated due to the coarser pixel resolution $\left(0.4^{\prime \prime}\right.$ per pixel) compared with the much finer Hubble Space Telescope (HST) resolution $\left(0.06^{\prime \prime}\right.$ per pixel). Bright fluxes in the NB images indicate the presence of strong emission lines.

IR and IRAC observations were taken as part of the GO12063 program (PI: S. Faber), and GO61042 program (PI: G. Fazio), respectively.

\subsection{Selection of $z=7.7$ Candidate Galaxies}

To generate the source catalog we first aligned all images that include HST/ACS images (F606W, F814W), near-IR NB1066 image (NB from DAWN survey), and near-IR images (F125W, F160W) onto a common world coordinate system grid. We then used a source detection software (SExtractor; Bertin \& Arnouts 1996) in dual image mode where the detection image (in this case the NB) is used to identify the pixels associated with each object, while the fluxes are measured from the respective photometry image.

For robust selection of candidate galaxies at $z=7.7$ we followed a set of criteria that has yielded a high spectroscopic success rate at $z=$ 4.5 and 5.7(Rhoads, \& Malhotra 2001; Rhoads et al. 2003; Dawson et al. 2004, 2007; Wang et al. 2009). Following this, each of our candidates had to satisfy all of the following criteria: 1) $5 \sigma$ detection in the NB filter, 2) $3 \sigma$ significant narrowband excess (compared to the F125W image), and 3) non-detection $(<2 \sigma)$ in the individual optical images (F606W, F814W). Criteria 1 \& 2 ensure real emission line sources while criterion 3 eliminates most low-redshift sources. Using this set of criteria we identified three $z=7.7$ candidates: $z 8 \_5$, z8_4, and z8_SM for spectroscopic follow-up. Formally, z8_SM has a NB S/N 4 (just below our selection threshold), however it has an aggregate $\mathrm{S} / \mathrm{N}=13$ in $\mathrm{NB}+\mathrm{F} 125 \mathrm{~W}+\mathrm{F} 160 \mathrm{~W}$. Given that it satisfied all other selection criteria, and given its 
proximity with other two galaxies, we included it for the spectroscopic follow-up observations. It should be noted that galaxy z8_5 was independently identified previously by Oesch et al (2015) using Lyman-break selection technique.

Figure 1 shows image cutouts of all three candidates in five filters. All three galaxies have significant fluxes in the NB filter, indicating the presence of strong emission lines, most likely Lyman- $\alpha$ lines at the observed wavelength of $1.066 \mu \mathrm{m}$. None of the galaxies have detectable fluxes at the visible wavelengths suggesting that these galaxies are consistent with being at redshifts $z \geq 7$. All three galaxies are detected in both F125W and F160W (see Table 1), despite two of them being faint, making z8_4 the faintest galaxy discovered at such high redshifts, thanks to the NB selection technique in which detection of galaxies does not depend on the continuum brightness.

\subsection{Photometric Redshifts And Spectral Energy Distributions}

To measure the photometric redshifts, we made use of spectral energy distribution (SEDs) templates. We obtained the best-fit SEDs using EAZY(Brammer et al. 2008) which provides photometric redshift probability distribution $p(z)$ by finding the best-fitting combination of redshifted galaxy spectral templates to the observed photometry. Because our galaxy sample is selected to have emission lines, to derive the best-fit SEDs and photometric redshifts, we chose a set of spectral templates that have emission lines (eazy_v1.3). These templates are corrected for the intergalactic absorption by neutral hydrogen, following the Madau prescription(Madau 1995). We allowed a wide range of redshift grids $(z=0.1$ to $z=9)$ to search for the best-fit SED template. In addition to the photometry (discussed in Section 2.2), we also used HST/WFC3 F105W photometry (GO:13792, PI: Bouwens) for z8_5 and CFHT-Y band photometry for z8_4 and z8_SM.
As shown in Figure 2, for all three galaxies the best-fit SEDs (shown in blue color) prefer spectral templates that correspond to a photometric redshift $z_{\text {phot }}=7.7$. This is also evident from the $p(z)$ (shown in the inset); the presence of strong emission lines in the NB filter yield very tight constraints on the $p(z)$. For completeness, we also show the low-redshift galaxy templates (grey color), which however are disfavored due to much larger $\chi^{2}$ values of the SED fit. For the brightest galaxy z8_5 (previously identified in Oesch et al 2015), the difference between best-fit $\chi^{2}$ value of low-redshift template and high-redshift template is 86 . For the remaining galaxies z8_4, and z8_SM while the difference between best-fit $\chi^{2}$ value of low-redshift template and high-redshift template is $>1$, it is not as high as for z8_5 given their lower S/N. Furthermore, all three galaxies have large restframe Lyman- $\alpha$ equivalent widths $\mathrm{EW}_{\text {rest }}>$ $23 \AA$ (see Table 1), which likely makes them visible as Lyman- $\alpha$ emitting galaxies.

\section{SPECTROSCOPIC OBSERVATIONS}

To confirm the photometric redshifts of these galaxies, we performed Y-band spectroscopy using Multi-Object Spectrometer for Infra-Red Exploration (MOSFIRE) spectrograph (McLean et al. 2012) on the Keck I telescope. The MOSFIRE instrument allows us to obtain spectroscopic observations of multiple objects simultaneously, with the Y-band covering Lyman- $\alpha$ lines redshifted to $z=7-8.2$. We targeted three Lyman- $\alpha$ galaxy candidates in the EGS field, as our primary science targets, and used low-redshift emission line candidates as fillers. Observations were taken on May 06, 2017, with each individual exposures having 140 sec integration time and $A B$ pattern dither offsets along the slit with offset of $\pm 1^{\prime \prime}$ from the center. The spectroscopic conditions were good with a typical seeing of $\sim 0.7^{\prime \prime}$, and a total integration time of about $4 \mathrm{hrs}$ per object. 
Table 1. Photometry and spectroscopic measurements of EGS77

\begin{tabular}{cccccccc}
\hline \hline ID & RA & DEC & F606W & F814W & NB & F125W & F160W \\
& J2000 & J2000 & mag & mag & mag & mag & mag \\
\hline z8_SM & $14: 20: 35.694$ & $+53: 00: 09.318$ & $<28.3^{1}$ & $<28.2^{1}$ & $24.76 \pm 0.35$ & $26.76 \pm 0.13$ & $26.66 \pm 0.11$ \\
z8_4 & $14: 20: 35.169$ & $+52: 59: 40.613$ & $<28.3^{1}$ & $<28.2^{1}$ & $23.85 \pm 0.15$ & $27.25 \pm 0.21$ & $27.10 \pm 0.16$ \\
z8_5 & $14: 20: 34.872$ & $+53: 00: 15.242$ & $<28.3^{1}$ & $<28.2^{1}$ & $23.60 \pm 0.12$ & $25.29 \pm 0.03$ & $25.08 \pm 0.03$ \\
\hline
\end{tabular}

Spectroscopic measurements

\begin{tabular}{ccccccccc}
\hline ID & $z_{\text {spec }}$ & $\begin{array}{c}f_{\text {Ly } \alpha} \\
\left(\mathrm{erg} \mathrm{s}^{-1} \mathrm{~cm}^{-2}\right)\end{array}$ & $\begin{array}{c}\mathrm{EW}_{\text {rest }} \\
\AA\end{array}$ & $\begin{array}{c}\mathrm{L}_{\text {Ly } \alpha} \\
10^{43}\left(\mathrm{erg} \mathrm{s}^{-1}\right)\end{array}$ & $\begin{array}{c}\text { HII radii } \\
\mathrm{pMpc}\end{array}$ & $\begin{array}{c}\text { SNR } \\
(\mathrm{Ly} \alpha)\end{array}$ & $\begin{array}{c}\text { Distance }^{3} \\
\text { Trans }\end{array}$ & LoS \\
\hline z8_SM & 7.767 & $0.29 \pm 0.06 \times 10^{-17}$ & $23 \pm 6$ & $0.2 \pm 0.1$ & 0.55 & 4.9 & 0.06 & 0.9 \\
z8_4 & 7.748 & $0.56 \pm 0.09 \times 10^{-17}$ & $71 \pm 18$ & $0.4 \pm 0.1$ & 0.69 & 6.0 & 0.09 & 0.2 \\
z8_5 & 7.728 & $1.7 \pm 0.14 \times 10^{-17}$ & $37 \pm 3$ & $1.2 \pm 0.1$ & 1.02 & 12.1 & 0.08 & -0.5 \\
\hline
\end{tabular}

Note $-{ }^{1} 2 \sigma$ magnitude limits. All magnitudes are AB magnitudes.

${ }^{2}$ HII bubble radii based on Fig 15 from Yajima et al 2018.

${ }^{3}$ Trans and LoS are the transverse and line-of-sight separation of each galaxy from the flux-weighted mean location of the group center, measured in pMpc.

We reduced data using the public MOSFIRE data reduction pipeline. It performs standard data reduction procedures including sky subtraction, rectification of the $2 \mathrm{D}$ spectra, and wavelength calibration. For a given object, it also produces corresponding signal-to-noise ratio (SNR) and a sigma image. The final 2D science spectra has a spatial resolution of $0.18^{\prime \prime}$ per pixel and a dispersion of $1.086 \AA$ per pixel. For absolute flux calibration of $1 \mathrm{D}$ spectra, we compared the measured line flux of z8_5 with the calibrated flux from Oesch et al. (2015), and converted instrumental flux (in counts) to the absolute flux. The conversion factor derived for z8_5 is then used to calibrate spectra of z8_SM and $\mathrm{z} 8 \_4$.

Figure 3 shows the final co-added twodimensional (2D) and one-dimensional (1D) Yband spectra of all three galaxies. As seen, both galaxies z8_5 and z8_4 show prominent emission lines $(\mathrm{S} / \mathrm{N}>5)$ while z8_SM has $\mathrm{S} / \mathrm{N}=4.9$. In the following we show that these are very likely Lyman- $\alpha$ emission at $z=7.7$.
For both galaxies z8_5 and z8_4, emission lines are free from the night sky $\mathrm{OH}$ lines, as shown in shaded region (middle row). For z8_SM, while its emission line is close to a faint night sky line, given its higher $\mathrm{S} / \mathrm{N}$ compared to the night sky lines, it is very likely a genuine emission line. Furthermore, the observed Y-position of the emission line in the 2D spectrum of z8_SM matches well with that expected based on the slit position in the mask (Fig 3 top panel), supporting our conclusion that this is a real emission line. We note that the presence of a faint night sky line at the position of the Lyman- $\alpha$ line may possibly affect the Lyman- $\alpha$ line flux measurements. However, given the faintness of the night sky line, we expect that it will have a minimal impact on the measurement of the Lyman- $\alpha$ line flux. In the following we demonstrate that these are very likely Lyman- $\alpha$ lines at $z=7.7$.

One of the characteristics that distinguishes Lyman- $\alpha$ emission from star-forming galaxies and other emission lines is the line asymme- 

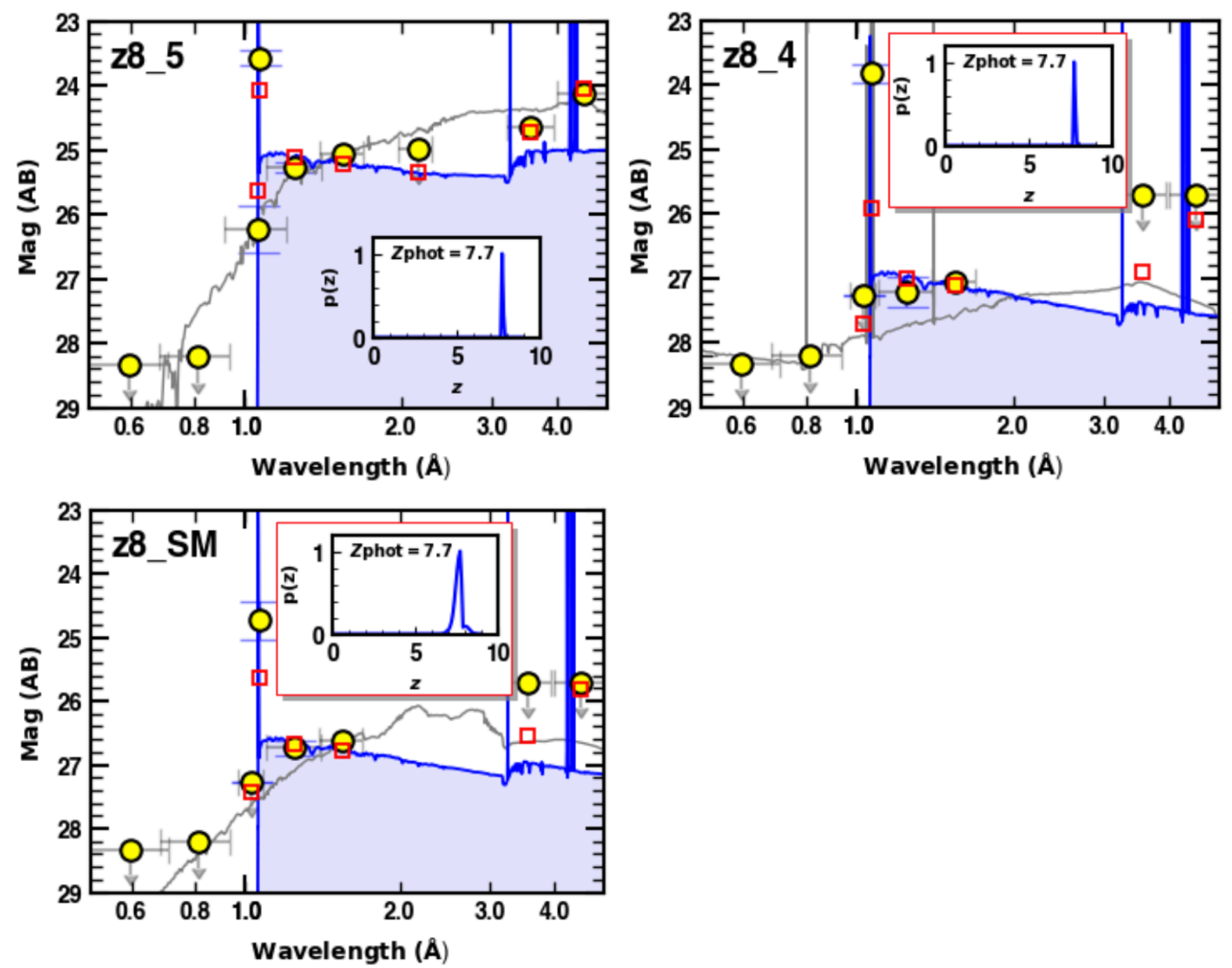

Figure 2. Spectral energy distributions (SEDs) of EGS77: SEDs based on fits to the photometry obtained from HST/ACS (F606W, F814W), NEWFIRM (NB), HST/WFC3 (F125W, F160W), and Spitzer IRAC (ch1, ch2). In addition, z8_5 photometry includes HST/WFC3 F105W observations while z8_SM and z8_4 include CFHT-Y band photometry. The blue line represents the best-fit spectral template while the filled yellow circles represent photometric observations. Open squares indicate best-fit template fluxes convolved with the respective filters. Circles with downward pointing arrows represent $2 \sigma$ non-detection limits. The best-fit photometric redshift distribution (shown in the inset) yields $z$ phot $=7.7$ which implies that there is a high probability of these galaxies being at high redshifts. For completeness, we also show low redshift galaxy spectral templates (shown in grey color) which are disfavored given their larger $\chi^{2}$ values. 

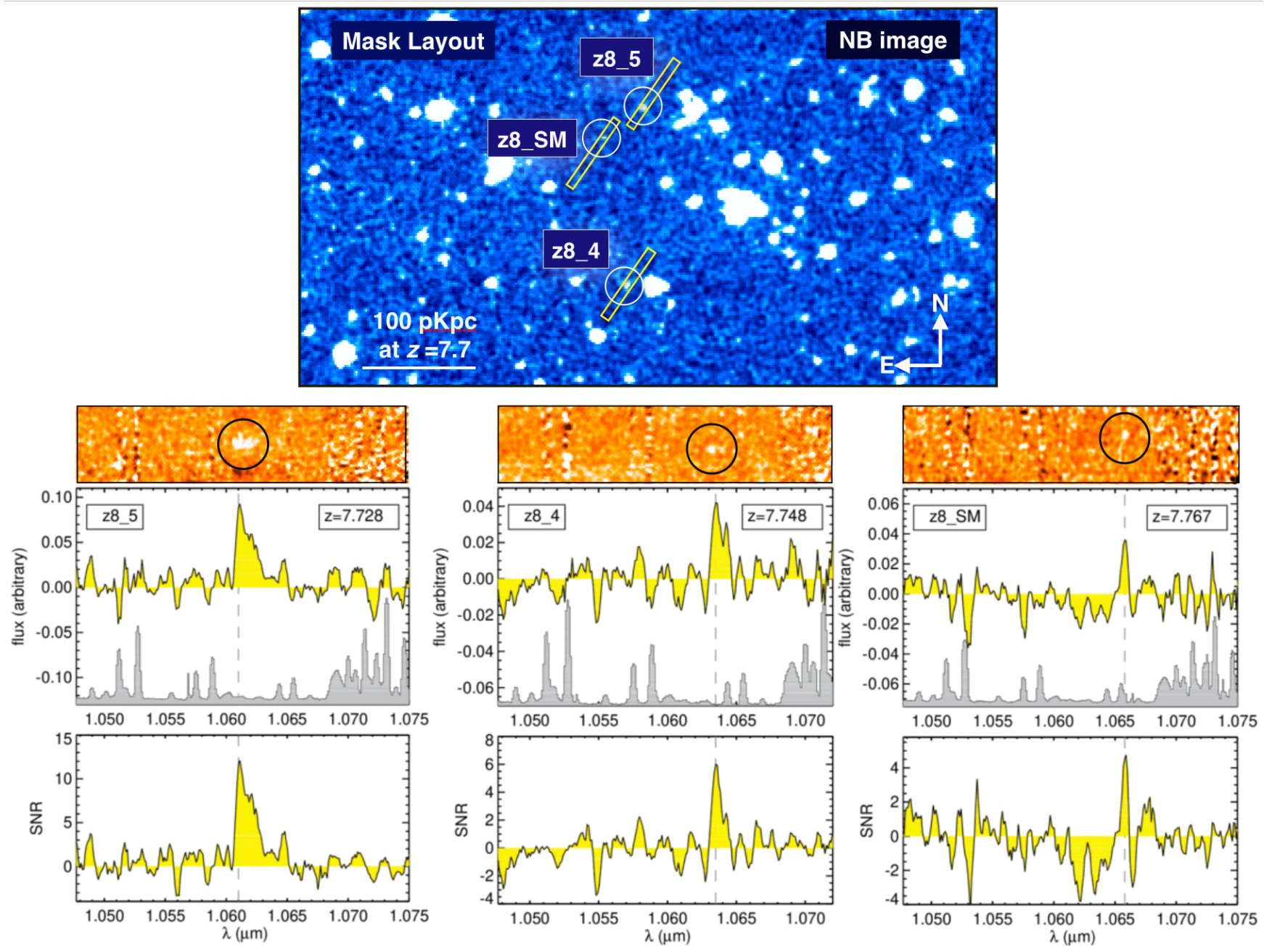

Figure 3. Spectroscopic observations of EGS77. Top: A portion of the Extended Groth Strip (EGS) field centered on EGS77, and the multi-object mask layout used for MOSFIRE Y-band spectroscopic observations. The mask is overlaid on the NB image $\left(\sim 2.3^{\prime} \times 1.2^{\prime}\right)$ where the image is slightly smoothed for clarity. Lower panels: Top and middle rows show final stacked $2 \mathrm{D}$ and $1 \mathrm{D}$ spectra respectively, while bottom row shows signal-to-noise ratio (SNR). The maximum of the SNR is normalized to the total SNR of the Lyman- $\alpha$ line. Shaded grey region shows night sky lines with arbitrary normalization. Lyman- $\alpha$ lines (shown by black circles, and represented with vertical dashed lines in the middle row) are detected in all three galaxies at the expected observed wavelength of about $1.066 \mu \mathrm{m}$.

try(Rhoads et al. 2003) or Skewness(Kashikawa et al. 2006). To quantify the Skewness in the line, we calculated the weighted skewness parameter $S w$, and found $S w=22 \pm 6 \AA$ for z8_5. This confirms that z8_5 is a $z=7.7$ galaxy because $S w>3 \AA$ is not seen in low-redshift emission lines of [OII], [OIII], and $\mathrm{H} \alpha$ (Kashikawa et al. 2006). Indeed z8_5 galaxy was previously identified as a Lyman break candidate (labelled as EGS-zs8-1), and spectroscopically confirmed as a $z=7.7$ galaxy(Oesch et al. 2015). Furthermore, recent H-band spectroscopic observations of this galaxy show presence of [CIII] 1909 doublet(Stark et al. 2017). Thus, given all the evidence, z8_5 is unequivocally a Lyman- $\alpha$ emitting galaxy at $z=7.7$. For galaxy z8_4 the weighted skewness parameter for the observed emission line is $S w=17 \pm 7 \AA$ confirming the line to be Lyman- $\alpha$ at $z=7.7$. For z8_SM, we can not reliably measure the asymmetry of the 
line given its lower $\mathrm{S} / \mathrm{N}$ in the spectrum. However, based on the best-fit SEDs, both z8_4 and z8_SM favor high redshift solutions. Furthermore, if these were faint, low-redshift galaxies, the best-fit low redshift SEDs implies a clear detection in F606W and F814W filters. Thus, given all the evidence, z8_5 and z8_4 are unequivocally at redshifts $z=7.728$ and $z=7.748$ respectively, and z8_SM is also very likely at redshift $z=7.767$.

It is striking that while z8_4 and z8_SM are faint ( $\left.\mathrm{M}_{\mathrm{UV}}>-20.3 \mathrm{mag}\right)$, both show Lyman$\alpha$ emission lines. Moreover, despite low number density of such faint galaxies at $z>7$, all three galaxies are spectroscopically confirmed. This high spectroscopic success rate is likely because (1) our NB technique preselects galaxies with detectably strong line emission, and (2) EGS77 is likely to have formed a large ionized bubble, allowing Lyman- $\alpha$ photons to escape. We discuss this in more detail in the following section.

\section{VISIBILITY OF LYMAN- $\alpha$}

The visibility of Lyman- $\alpha$ emission from starforming galaxies at high redshifts depends on several factors including star-formation rate, ionizing photon budget, galactic outflows, and the density of neutral hydrogen surrounding the galaxy. The star-formation rate and the ionizing photon budget will directly influence the amount of ionized gas forming an ionized bubble which in turn allows Lyman- $\alpha$ photons to travel unattenuated along the line-of-sight(Malhotra, \& Rhoads 2006).

The separation between the most distant member z8_SM $(z=7.767)$ and $z 8 \_4(z=$ 7.748) along the line-of-sight is about $0.7 \mathrm{pMpc}$ (physical Mpc), which is same as the separation between z8_4 and z8_5 $(z=7.728)$. In the transverse direction (i.e. projection on the sky) all three members are much closer to each other. The separation between z8_SM and z8_5 in the transverse direction is $10^{\prime \prime}(0.05$ pMpc), while the separation between z8_4 and z8_5 is $35^{\prime \prime}(0.18 \mathrm{pMpc})$. Thus, given the proximity of all three galaxies with each other in both the transverse direction and along the line-of-sight, these galaxies will form a continuous attenuation-free path for Lyman- $\alpha$ photons if the radii of their ionized bubbles are $\geq 0.35 \mathrm{pMpc}$ along the line-of-sight direction (Fig. 4). This is because the ionized region is large enough that the Lyman- $\alpha$ photons are redshifted by the time they reach the neutral hydrogen boundary, and thus can escape. This is supported by recent spectroscopic observations of the brightest galaxy z8_5, showing a [CIII] 1909 doublet(Stark et al. 2017), which yields a velocity offset $\Delta V_{L y \alpha}=340_{-30}^{+15} \mathrm{~km} \mathrm{~s}^{-1}$. When compared to a $F W H M=360 \mathrm{~km} \mathrm{~s}^{-1}$ for this line(Oesch et al. 2015), it implies that a substantial fraction of Lyman- $\alpha$ photons are leaving the galaxy at $340-520 \mathrm{~km} \mathrm{~s}^{-1}$.

\subsection{Estimation of Bubble Sizes}

We now estimate the sizes of ionized bubbles formed by these galaxies, based on theoretical model where the relation between Lyman- $\alpha$ luminosities and bubble sizes has been predicted through simulations, while the star formation rates is derived using growth rate of halo mass with a constant tuning parameter (Yajima et al. 2018). The growth rate of halos is calculated using the halo merger trees based on an extended Press-Schechter formalism (Somerville, \& Kolatt 1999; Khochfar, \& Burkert 2001). These simulations reproduce the observed star formation rate density as well as the UV luminosity function of Lyman break selected galaxies at $z \sim 7$ and $z \sim 8$ (Bouwens et al. 2012). Next, to estimate the sizes of ionized bubble which is proportional to the ionizing photon budget, they use star formation history of each halo using stellar population synthesis code STARBURST99 (Leitherer et al. 1999). Finally, the Lyman- $\alpha$ luminosity of each galaxy is calculated based on the number of ionizing photons absorbed within the galaxy. Photons that are 


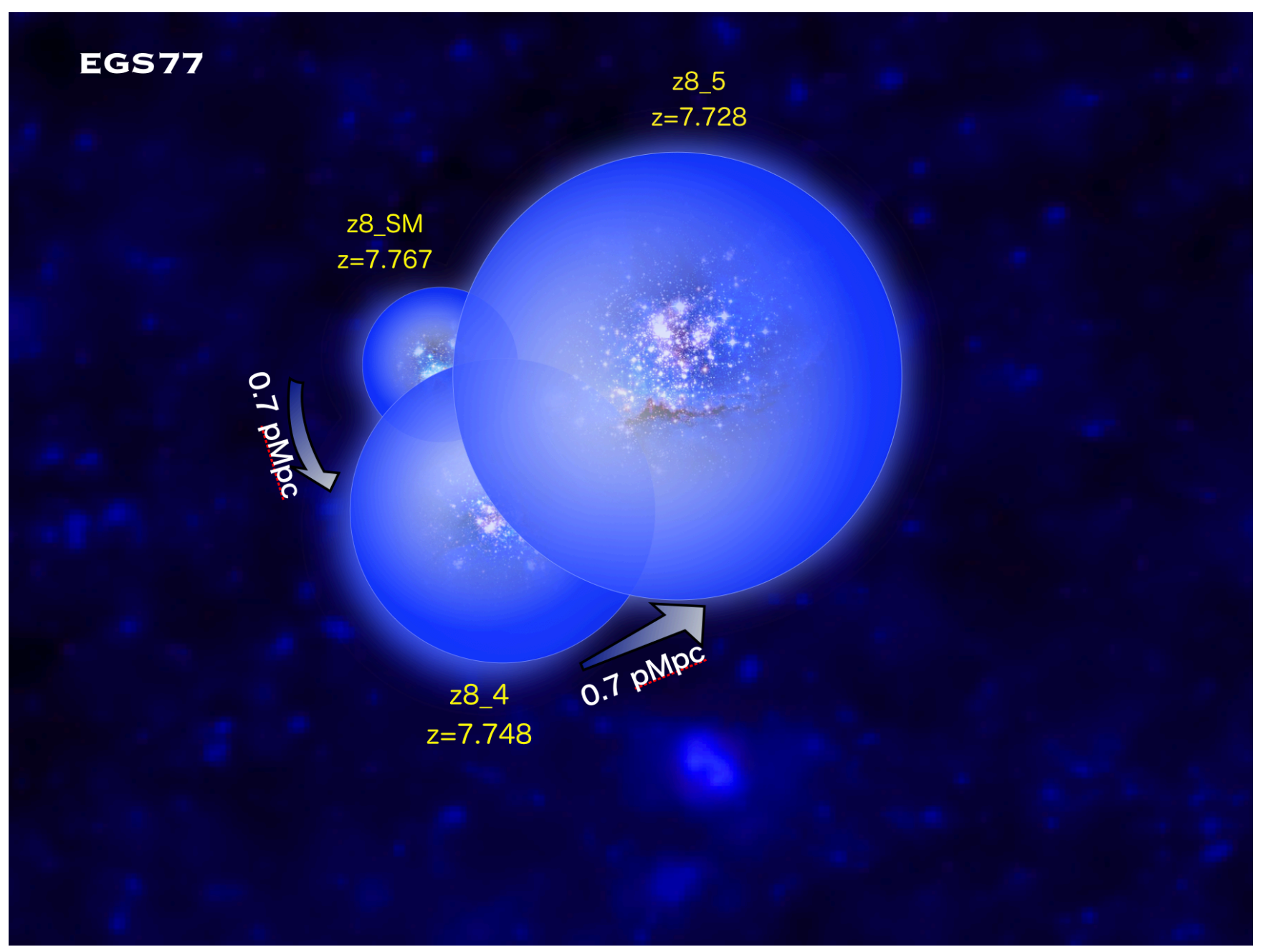

Figure 4. Rendition of the galaxy group EGS77: Rendition showing ionized bubbles formed by three galaxies near redshift 7.7. Galaxy z8_5 is the brightest of all three, with the largest ionized bubble, and lies in the front. It is estimated to produce an ionized bubble of radius $1 \mathrm{pMpc}$, which will help clear the way for transmission of Lyman- $\alpha$ photons from the two fainter galaxies. The physical separation between each pair of galaxies is merely $0.7 \mathrm{pMpc}$ along the line of sight, which implies that their ionized bubbles overlap substantially to create a continuous path allowing Lyman- $\alpha$ photons to escape.

absorbed will produce Lyman- $\alpha$ photons while photons that escape cause the cosmic reionization. Based on these simulations (Figure 15 from Yajima et al 2018), the luminosity of our brightest galaxy z8_5 yields a radius of 1.02 pMpc for the HII bubble. For the remaining two galaxies z8_4 and z8_SM we get the bubble sizes of 0.69 and $0.55 \mathrm{pMpc}$ respectively (see Fig 4). This implies that the ionized bubbles produced by individual galaxies overlap significantly, forming a continuous non-attenuating path through which Lyman- $\alpha$ photons can eas- ily escape and hence be observed(Larson et al. 2018). This is currently the only observational evidence of an ionized bubble formed by a group of three galaxies (EGS77) during the onset of cosmic reionization. This localized ionized region leads to the expected spatial inhomogeneity in the reionzation process, and provides evidence of fainter galaxies' contribution to this process.

\section{SUMMARY}


In this Letter we reported the discovery of the farthest galaxy group EGS77 at $z=7.7$, merely 680 Myrs after the Big Bang. EGS77 was initially identified using NB imaging observations from the DAWN survey, and later confirmed via spectroscopic observations using MOSFIRE spectrograph on the Keck telescope. It is striking that all three galaxies in EGS77 are spectroscopically confirmed via Lyman- $\alpha$ emission line $\left(\mathrm{SNR}_{\mathrm{Ly} \alpha} \gtrsim 5\right)$ despite two of them being faint in the continuum. In fact EGS77 contains the faintest galaxy (in terms of the continuum brightness) discovered and spectroscopically confirmed at this redshift. Based on model and simulations from literature, we found that the ionized bubbles produced by all three galaxies overlap significantly, producing a large, yet localized ionized region giving rise to the expected inhomogeneity in the reionization process. Thus, this is the first observations of a galaxy group responsible for the cosmic reionization. The James Webb Space Telescope will be sensitive to the rest-frame UV continuum of such fainter galaxies in the reionization epoch, and therefore can provide a much larger sample of galaxies responsible for the re-ionization process. Furthermore, future $21 \mathrm{~cm}$ observations from SKA-2(Dewdney et al. 2009) will be able to probe the ionized structures with angular size as large as $\sim 10^{\prime}$, similar to the size of ionized bubble produced by z8_5 at $z=7.7$.

\section{ACKNOWLEDGMENTS}

We thank the US National Science Foundation (NSF Grant AST-1518057), and NASA for its financial support through the WFIRST Science Investigation Team program (NNG16PJ33C). Z.Y.Z. thanks NSF of China (11773051) and the CAS Pioneer Hundred Talents Program. Data presented herein were obtained at the W.M. Keck Observatory, which is operated as a scientific partnership among Caltech, the University of California and the NASA. The Observatory was made possible by the generous financial support of the W.M. Keck Foundation. The authors wish to recognize and acknowledge the very significant cultural role and reverence that the summit of Maunakea has always had within the indigenous Hawaiian community. We are most fortunate to have the opportunity to conduct observations from this mountain.

\section{REFERENCES}

Bertin, E., \& Arnouts, S. 1996, A\&AS, 117, 393

Brammer, G. B., van Dokkum, P. G., \& Coppi, P. 2008, ApJ, 686, 1503

Bouwens, R. J., Illingworth, G. D., Oesch, P. A., et al. 2012, ApJ, 754, 83

Bouwens, R. J., Illingworth, G. D., Oesch, P. A., et al. 2015, ApJ, 811, 140

Coughlin, A., Rhoads, J. E., Malhotra, S., et al. 2018, ApJ, 858, 96

Dawson, S., Rhoads, J. E., Malhotra, S., et al. 2004, ApJ, 617, 707

Dawson, S., Rhoads, J. E., Malhotra, S., et al. 2007, ApJ, 671, 1227

Dewdney, P. E., Hall, P. J., Schilizzi, R. T., et al. 2009, IEEE Proceedings, 97, 1482

Hu, E. M., Cowie, L. L., Barger, A. J., et al. 2010, ApJ, 725, 394
Kashikawa, N., Shimasaku, K., Malkan, M. A., et al. 2006, ApJ, 648, 7

Khochfar, S., \& Burkert, A. 2001, ApJ, 561, 517

Larson, R. L., Finkelstein, S. L., Pirzkal, N., et al. 2018, ApJ, 858, 94

Leitherer, C., Schaerer, D., Goldader, J. D., et al. 1999, ApJS, 123, 3

Madau, P. 1995, ApJ, 441, 18

Malhotra, S., \& Rhoads, J. E. 2006, ApJL, 647, L95

Oesch, P. A., van Dokkum, P. G., Illingworth, G. D., et al. 2015, ApJL, 804, L30

Ouchi, M., Shimasaku, K., Furusawa, H., et al. 2010, ApJ, 723, 869

McLean, I. S., Steidel, C. C., Epps, H. W., et al. 2012, Proc. SPIE, 84460J 
Probst, R. G., Gaughan, N., Abraham, M., et al. 2004, Proc. SPIE, 1716

Probst, R. G., George, J. R., Daly, P. N., et al. 2008, Proc. SPIE, 70142S

Rhoads, J. E., \& Malhotra, S. 2001, ApJL, 563, L5

Rhoads, J. E., Hibon, P., Malhotra, S., et al. 2012, ApJL, 752, L28

Rhoads, J. E., Dey, A., Malhotra, S., et al. 2003, AJ, 125, 1006

Somerville, R. S., \& Kolatt, T. S. 1999, MNRAS, 305,1

Stark, D. P., Ellis, R. S., Charlot, S., et al. 2017, MNRAS, 464, 469
Steidel, C. C., Adelberger, K. L., Shapley, A. E., et al. 2003, ApJ, 592, 728

Stiavelli, M., Fall, S. M., \& Panagia, N. 2004, ApJL, 610, L1

Swaters, R. A., Valdes, F., \& Dickinson, M. E. 2009, Astronomical Data Analysis Software and Systems XVIII, 506

Wang, J.-X., Malhotra, S., Rhoads, J. E., Zhang, H.-T., \& Finkelstein, S. L. 2009, ApJ, 706, 762

Yajima, H., Sugimura, K., \& Hasegawa, K. 2018, MNRAS, 477, 5406 